\title{
Developmental susceptibility to the horizontal-vertical illusion
}

\author{
GARY M. BROSVIC, STACEY BAILEY, ANNE BAER, and JODI ENGEL \\ Rider College, Lawrenceville, New Jersey \\ ROBERTA E. DIHOFF and LARA CARPENTER \\ Rowan College, Glassboro, New Jersey \\ SHERRY BAKER \\ Rider College, Lawrenceville, New Jersey \\ and \\ MICHAEL COOK \\ Rowan College, Glassboro, New Jersey
}

\begin{abstract}
Developmental susceptibility to four forms of the horizontal-vertical (HV) illusion was examined in subjects ranging in age from 3 to 20 years. For each form of the HV illusion, one standard and six comparison stimuli were developed, with subjects first inspecting the standard and then selecting the matching comparator. The magnitude of the illusion, estimated from the difference between the size of the standard and that of the selected comparator, was found to be similar to values reported previously and determined through the use of a wide range of stimuli, procedures, and subject groups. Susceptibility to the HV illusion was greatest for the youngest subjects. It decreased for older subjects in accordance with the development of spatial coordinates, as suggested by Piaget.
\end{abstract}

The issue of developmental susceptibility to geometric illusions is controversial. Beginning with the observations of Binet (1895), susceptibility to the Müller-Lyer (ML), Sander parallelogram (SP), and horizontal-vertical (HV) illusions has been reported as decreasing with subjects' age, prompting the inclusion of these stimuli in early test batteries for developmental trends (Dawson, Young, \& Choi, 1973) and cultural differences in subjects' responsivity to visual stimuli (Rivers, 1901a, 1901b, 1905).

In most recent studies of the HV illusion, susceptibility to the L-shaped and inverted-T figures has been reported as declining in subjects between the ages of 6 and 11 (Dawson et al., 1973; Walter, 1942; Wursten, 1947). For the ML and SP illusions, similar declines have been reported between the ages of 3 and 12 (Binet, 1895; Dawson et al., 1973; Piaget \& Albertini, 1954; Walter, 1952; Wapner \& Werner, 1957). These trends, however, have not been observed in other studies (Grieve, Hogben, \& Williams, 1983), or they have been attributed to cultural differences (Segall, Campbell, \& Herskovits, 1963, 1966), eye pigmentation (Berry, 1972; Pollack \& Silver, 1967), stimulus figure color (Pollack, 1970), type of stimulus figure (L-shaped vs. inverted-T figure; see Fraisse \& Vautrey, 1956), or the complexity of experimental procedures (Grieve et al., 1983).

Correspondence should be addressed to G. M. Brosvic, Biopsychology Laboratory, Department of Psychology, Rider College, 2083 Lawrenceville Rd., Lawrenceville, NJ 08648-3099.
In prior studies of the HV illusion, the magnitude of the illusion has typically been greater with adjustments of the inverted-T figure than with adjustments of an Lshaped figure (Avery \& Day, 1969; Cormack \& Cormack, 1974; Kunnapas, 1955; McBride, Risser, \& Slotnick, 1987). These results have been interpreted as underscoring the importance of line bisection and a comparison line in the formation of the HV illusion, although the potentially confounding effects of line bisection on children's performance are unknown (Wohlhill, 1960). In prior studies in our laboratory, we have shown that the HV illusion can be readily demonstrated in the absence of line bisection and a comparison line. Indeed, when subjects are instructed to produce 1-in. vertical and horizontal lines, the magnitude of the illusion for vertical lines is greater than that for horizontal lines and does not differ from that for adjustments of the vertical line of the inverted-T figure (Brosvic \& Cohen, 1988; Brosvic et al., 1993; McBride et al., 1987).

The psychophysical procedures in many studies of visual illusions require subjects to make multiple judgments of test stimuli, and to do so for an extended period. The measurement of an adult's susceptibility to illusions can be accomplished through the use of such methods, although the use of similar procedures with children may be questioned, especially since children up to the age of 10 may not understand the directions of adults (Chomsky, 1969; Palermo \& Molfese, 1972), may answer randomly to mask their lack of understanding (Abbeduto, 1985; 
Carey, 1978; Grieve, 1981; Grieve et al., 1983; Hughes \& Grieve, 1980; Terrell, 1991), and, independently of the experimental instructions, may also simply select the larger part of an array when they respond (Braine \& Shanks, 1965).

The results of pilot studies conducted in our laboratory led us to develop a common set of procedures for studying four forms of the HV illusion; we used them to examine the issue of developmental susceptibility to the HV illusion in subjects ranging in age from 3 to 20 years.

\section{METHOD}

\section{Subjects}

Ten subjects at each age ranging from 3 to 10 years and 5 subjects at each age ranging from 11 to 20 years served as subjects. Each subject reported normal or corrected visual acuity.

\section{Stimuli and Procedures}

The test stimuli consisted of modified versions of the paper-and-pencil adjustment (L-shaped and inverted-T figures) and production task (vertical and horizontal lines) forms of the HV illusion described previously (Brosvic \& Cohen, 1988), and in each instance, one standard and six comparison stimuli were developed.

Inverted-T and $\mathbf{L}$-shaped figures. The standard stimulus for the inverted-T figure consisted of a 2 -in.-long vertical line bisecting a 2 -in.long horizontal line. In the comparison stimuli, one figure was identical in length to the standard; in three others, the vertical line had been increased in length by $10 \%, 20 \%$, or $30 \%$; and in the remaining three figures, the length of the vertical line had been decreased in length by $10 \%, 20 \%$, or $30 \%$. The length of the horizontal line was always 2 in. The standard stimulus for the L-shaped figure also consisted of a 2-in. vertical line and a 2 -in. horizontal line, although the lines were not bisected. The comparison stimuli were identical in length to those described for the inverted-T figure, and the width of all stimulus lines was $1 \mathrm{~mm}$.

Vertical and horizontal lines. Either a 2-in.-long vertical or a 2 -in. horizontal line was printed in the center of each page to serve as the standard stimulus. For the comparison stimuli, one line was identical in length to the standard, three others were reduced in length by $10 \%$, $20 \%$, or $30 \%$, and the remaining three lines were increased in length by $10 \%, 20 \%$, or $30 \%$. The width of each vertical and horizontal line was $1 \mathrm{~mm}$.

Test procedures. For each form of the HV illusion described above, the standard was presented for $30 \mathrm{sec}$, removed from sight, and then replaced by a single sheet of paper on which the six comparators and the standard were printed. The subjects were then instructed to select the figure "that was the same" as the one they had initially inspected; that is, a correct response consisted of selecting the standard stimulus. The subjects completed five trials for each standard stimulus and did so in accordance with a Latin-square design. The magnitude of illusion for each form of the HV illusion was estimated by averaging the size difference between the standard and the selected comparator (range, -30 to 30). Potential differences within each form of the HV illusion as a function of age, as well as those between the four forms of the HV illusion, were examined with analysis of variance.

Pilot tests were conducted with the stimuli and procedures described above, and the instructions were found to be easily understood and completed by subjects as young as age 3 , with similar results obtained in follow-up tests in which children visually inspected an object printed on paper and then selected the same object from a comparison group. It should be noted that although the measurements made in this study are not as refined as those determined with the PEST procedure described by Grieve et al. (1983), they do appear to have met the criteria of being "clinical and concentric."

\section{RESULTS}

No differences in the estimated magnitude of illusion were observed as a function of sex of subject for any form of the HV illusion, so the data were combined across sexes for subsequent analyses [all $t \mathrm{~s}<1.32$, all $p s>.05$ ].

\section{Inverted-T Figure}

As can be seen in Figure 1, the subjects below the age of 11 were the most susceptible to the inverted-T form of the HV illusion $[F=26.43, p<.05]$, after which a relatively asymptotic level of performance was observed. Figure 1 indicates that the illusion was also evident, although at a reduced level, for the oldest subjects.

\section{L-Shaped Figure}

As can be seen in Figure 2, the subjects below the age of 9 were the most susceptible to the L-shaped form of the HV illusion $[F=17.29, p<.05]$, after which a relatively asymptotic level of performance was observed.

\section{Vertical Line}

Although the presence of line bisection and a comparison line contribute to the HV illusion, the illusion was apparent in their absence. As can be seen in Figure 3, the subjects below the age of 14 were the most suscepti-

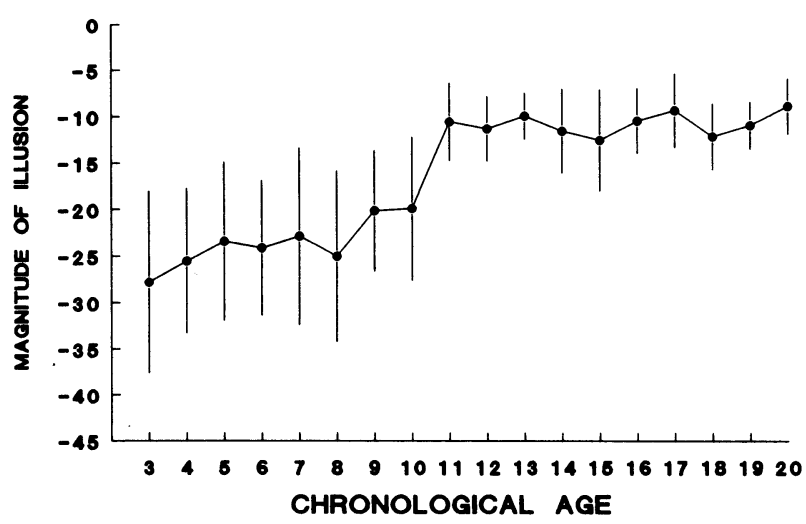

Figure 1. Estimated magnitude of illusion for the inverted-T figure as a function of age of subject. (Susceptibility declines after age 13.) The error bars represent $\pm 1 S D$.

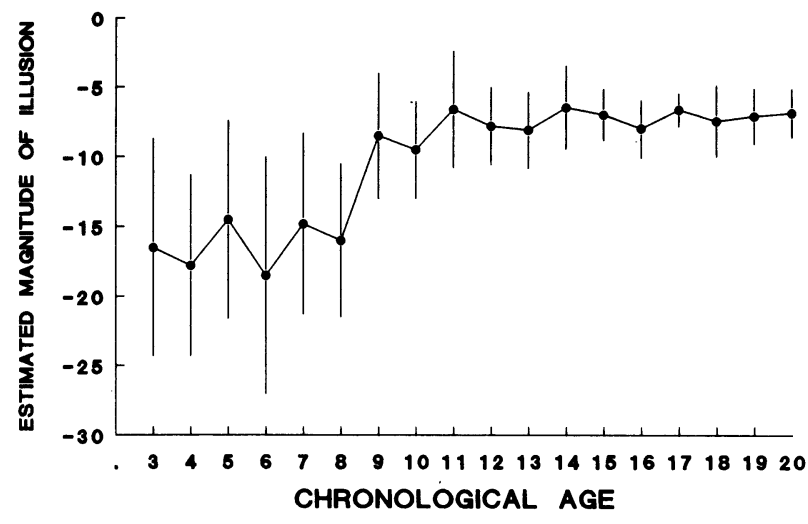

Figure 2. Estimated magnitude of illusion for the $\mathbf{L}$-shaped figure as a function of age of subject. (Susceptibility declines after age 8.) The error bars represent $\pm 1 S D$. 


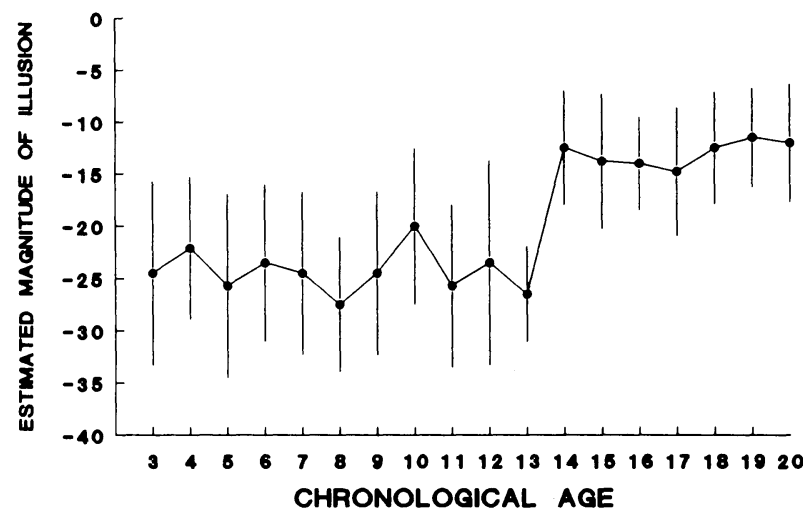

Figure 3. Estimated magnitude of illusion for the vertical line figure as a function of age of subject. (Susceptibility declines after age 13.) The error bars represent $\pm 1 S D$.

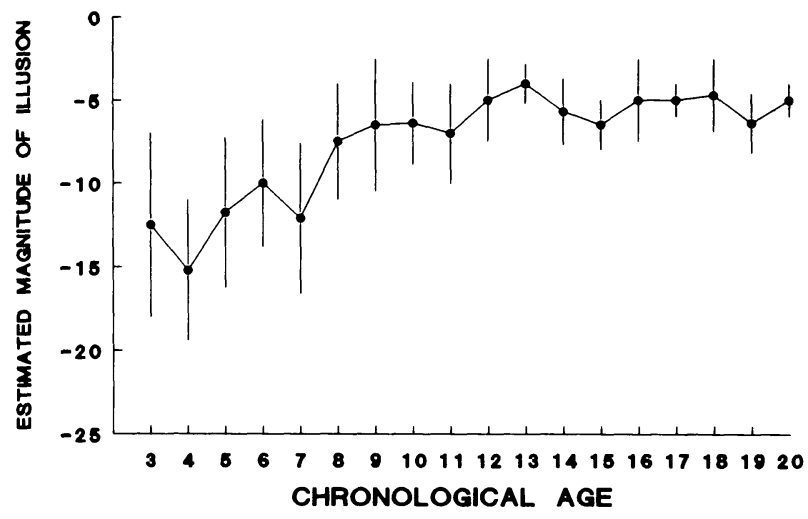

Figure 4. Estimated magnitude of illusion for the horizontal line figure as a function of age of subject. (Susceptibility declines after age 7.) The error bars represent $\pm 1 S D$.

ble to the vertical line form of the HV illusion $[F=47.08$, $p<.05]$, after which a relatively asymptotic level of performance was observed. Figure 3 indicates that the illusion was also evident, although at a reduced level, for the oldest subjects.

\section{Horizontal Line}

As can be seen in Figure 4, the subjects below the age of 8 were the most susceptible to the horizontal line form of the HV illusion $[F=17.29, p<.05]$, after which a relatively asymptotic level of performance was observed.

\section{DISCUSSION}

The present results, found with subjects ranging in age from 3 to 20 years, support prior observations that the magnitude of the illusion is greater for the inverted-T than for the L-shaped figure (Avery \& Day, 1969; Brosvic \& Cohen, 1988; Brosvic et al., 1993; Kunnapas, 1955; McBride et al., 1987). Although this finding highlights the role of line bisection in the formation of the HV illusion, the magnitude of the illu- sion for the vertical line and the inverted-T figure did not differ, suggesting that the HV illusion exists in the absence of line bisection and a comparison line.

It should be noted that the magnitude of the illusion for each task was estimated by the difference between the size of the standard stimulus and that of the selected comparator, and that the potential effects of recognition memory on the present results should be examined in future studies. The estimated magnitudes of the illusion observed in the present study are similar to previously reported values found through the use of a wide range of stimuli, procedures, and subject groups. Although the noteworthy differences among these procedures and stimuli preclude the direct numerical comparison of results, the estimates of magnitude illusion observed in the present study are similar to those reported by Dawson et al. (1973), Doyle (1967), Piaget, Matalon, and Vinh-Bang (1961), and Walter (1942). The developmental trends observed in the present study are also similar to those described previously for the ML and SP illusions (see Dawson et al., 1973).

The developmental trends observed in the present study most likely represent the gradual development of the ability to process spatial coordinates (Piaget \& Morf, 1956), and, even at an early age, this processing system appears to be sensitive to the different forms of the HV illusion. Piaget and Morf reported that the intersection of lines in the inverted-T figure provides a unique spatial framework that is not a property of the $\mathrm{L}$ shape, which could explain the larger magnitude of illusion observed for the inverted-T figure, the earlier age at which susceptibility to the $\mathrm{L}$ shape decreases, and the robustness of the response to the inverted-T figure observed in older subjects.

\section{REFERENCES}

Abreduto, L. (1985). The effects of linguistic complexity on children's and adults' motor programming of speech. Language \& Speech, 28, 361-375.

Avery, G. C., \& DAY, R. H. (1969). Basis of the horizontal-vertical illusion. Journal of Experimental Psychology, 81, 376-380.

BERRY, J. W. (1972). Müller-Lyer susceptibility: Culture, ecology, or race? International Journal of Psychology, 6, 193-197.

BINET, A. (1895). La mesure des illusions visuelles chez les enfants. Revue Philosophique de France et de l'Etranger, 40, 11-25.

Braine, M. D., \& Shanks, B. L. (1965). The development of conservation of size. Journal of Verbal Learning \& Verbal Behavior, 4, 227-242.

Brosvic, G. M., \& CoHen, B. D. (1988). The horizontal-vertical illusion and knowledge of results. Perceptual \& Motor Skills, 67, 463-469.

Brosvic, G. M., Farrelly, M., Rebele, E., Ribardo, D., GutowsKı, J., KAFER, L., \& DiHOFF, R. E. (1993). Nonequivalent roles for motor and visual feedback in the Müller-Lyer and horizontal-vertical illusions. Bulletin of the Psychonomic Society, 31, 42-44.

Carey, S. (1978). 'Less' never means 'more.' In R. N. Campbell \& P. T. Smith (Eds.), Recent advances in the psychology of language: Vol. 1. Language development and mother-child interaction (pp. 120 135). London: Plenum.

СномSкY, С. (1969). The acquisition of syntax in children from 5 to 10. Cambridge, MA: MIT Press.

Cormack, E. O., \& Cormack, R. H. (1974). Stimulus configuration and line orientation in the horizontal-vertical illusion. Perception \& Psychophysics, 16, 208-212.

Dawson, J. L., Young, B. M., \& ChOI, P. C. (1973). Developmental influences on geometric illusion susceptibility among Hong Kong Chinese children. Journal of Cross-Cultural Psychology, 4, 49-74.

Doyle, M. (1967). Perceptual skill development: A possible resource for the intellectually handicapped. American Journal of Mental Deficiency, 71, 776-782.

Fraisse, P., \& Vautrey, P. (1956). The influence of age, sex and specialized training on the vertical-horizontal illusion. Quarterly Journal of Experimental Psychology, 8, 114-120.

Grieve, R. (1981). Observations on communication problems in normal children. In W. I. Fraser \& R. Grieve (Eds.), Communicating with normal and retarded children (pp. 204-234). Bristol, U.K.: Wrights. 
Grieve, R., Hogben, J., \& Williams, A. (1983). Measurement of developmental susceptibility to the horizontal-vertical illusion. British Journal of Developmental Psychology, 1, 155-161.

Hughes, M., \& Grieve, R. (1980). On asking children bizarre questions. First Language, 1, 149-160.

KunNapas, T. (1955). An analysis of the "vertical-horizontal illusion." Journal of Experimental Psychology, 49, 134-140.

McBride, S., Risser, J. M., \& Slotnick, B. M. (1987). The horizontalvertical illusion: Independence of line bisection and a comparison line. Perceptual \& Motor Skills, 64, 943-948.

PAlermo, D. S., \& Molfese, D. L. (1972). Language acquisition from age five onward. Psychological Bulletin, 78, 409-428.

Piaget, J., \& Albertini, B. (1954). Recherches sur le development des perceptions, XIX. Observations sur la perception des bonnes formes dans l'enfant par actualisation des lignes virtuelles. Archives de Psychologie, 34, 203-243.

Piaget, J., Matalon, B., \& Vinh-Bang, K. (1961). Recherches sur le development des perceptions: XLII. L'évolution d'illusion dite 'verticale-horizontale'de ses composantes (rectangle et équerre) et de l'illusion de Delboeuf en presentation tachistoscopique. Archives de Psychologie, 38, 23-68.

Piaget, J., \& MoRf, A. (1956). Recherches sur la development des perceptions, XXX. Les comparaisons verticales a faible intervalle. Archives de Psychologie, 35, 289-319.

Pollack, R. H. (1970). Müller-Lyer illusion: Effect of age, lightness contrast and hue. Science, 170, 93-95.

Pollack, R. H., \& Silvar, S. D. (1967). Magnitude of the MuellerLyer illusion in children as a function of pigmentation of the fundus oculi. Psychonomic Science, 8, 83-84.
RIvERS, W. H. (1901a). Introduction and vision. In A. C. Haddon (Ed.), Reports of the Cambridge anthropological expedition to the Torres Straits (pp. 48-61). Cambridge: Cambridge University Press.

Rivers, W. H. (1901b). Primitive color vision. Popular Science Monthly, 59, 44-58.

Rivers, W. H. (1905). Observations on the senses of the Todas. British Journal of Psychology, 1, 321-396.

Segall, M. H., Campbell, D. T., \& Herskovits, M. J. (1963). Cultural differences in the perception of geometric illusions. Science, 139 , 769-771.

Segall, M. H., Campbell, D. T., \& Herskovits, M. J. (1966). The influence of culture on visual perception. Indianapolis, IN: Bobbs-Merrill.

TERRELL, T. D. (1991). The role of grammar instruction in a communicative approach. Modern Language Journal, 75, 52-63.

WALTER, A. (1942). A genetic study of geometrical-optical illusions. Genetic Psychology Monographs, 25, 101-155.

WaPNer, S., \& Werner, H. (1957). Perceptual development: An investigation within the framework of sensory-tonic field theory. Worcester, MA: Clark University Press.

Wohlhill, J. F. (1960). Developmental studies of perception. Psychological Bulletin, 57, 249-288.

WURSTEN, H. (1947). Recherches sur le development des perceptions, IX. L'évolution des comparaisons de longueris de l'enfant à l'adulte avec variation d'angle entre la verticale et l'horizontale. Archives Psychology (Genève), 32, 1-144.

(Manuscript received July 3, 1993.) 